\title{
CHEMICAL, OPTICAL, AND INFRARED PROPERTIES OF QUENCHED CARBONACEOUS COMPOSITES (GCCS)
}

\author{
AKIRA SAKaTA \\ Department of Applied Physics and Chemistry, University of \\ Electro-Communications \\ SETSUKO WADA \\ Laboratory of Chemistry, University of Electro-Communications
}

\begin{abstract}
Quenched carbonaceous composite (QCC) was synthesized by quenching the plasma of methane gas. Chemical properties as well as optical and infrared spectra of the QCC and "oxidized" QCC were measured. Good agreement of the IR spectra of the QCCs to the unidentified infrared (UIR) emission bands was obtained. Correspondence of their features to molecular structures in the QCC was estimated. It is concluded that a "cross-conjugated ketone" structure (CCK) caused the 6.2, 7.7, and $8.6 \mu \mathrm{m}$ features and "solo" $H$ atoms on carbon are responsible for the 3.3 and $11.3 \mu \mathrm{m}$ features.
\end{abstract}

\section{INTRODUCTION}

We have reported that a carbonaceous composite synthesized from a hydrocarbon plasma (Quenched Carbonaceous Composite: QCC) shows extinction properties closely resembling the observed average interstellar extinction curve around the 220 nm hump (Sakata et al., 1983) and IR features at 3.3, 3.4, 3.5, 6.2, 6.9, 7.3, 7.7, 8.6, 11.4, 11.9, and $13.2 \mu \mathrm{m}$ corresponding to the observed unidentified IR bands (Sakata et al., 1984, 1987). This paper summarizes work presented in these papers and presents new data on the spectra of QCC at high temperature.

Two types of circumstellar dust formation are possible corresponding to equilibrium and non-equilibrium processes. The $\mathrm{QCC}$ is a product formed from a nonequilibrium process. In this paper we will discuss the formation process of QCC, its optical properties and the correlation of the optical properties to observed data.

\section{EXPERIMENTAL}

Our experimental apparatus is composed of five parts:

1. gas supply system, 
2. plasma chamber,

3. spectrometer system for plasma radiation,

4. vacuum reaction chamber, and

5. analyser system for the produced gases.

The experimental conditions are as follows: the starting gas is methane at a pressure of 4 Torr. A plasma is generated and maintained in the chamber with a $300 \mathrm{~W}$ microwave generator.

\section{RESULTS}

\subsection{PLASMA DIAGNOSTICS}

Spectroscopy of the plasma gas showed intense emission lines of radicals and atoms, such as $\mathrm{CH}, \mathrm{C}_{2}$, and $H$. The electronic excitation temperature of the hydrogen atoms was determined to be about $6000 \mathrm{~K}$ from the intensity ratio of $\mathrm{H}_{\alpha}$ and $\mathrm{H}_{\beta}$. Analysis of the Swan band of $C_{2}$ indicated a vibrational temperature of about 3000 $\mathrm{K}$. The rotational temperature of $C H$ was obtained from the $4300 \AA$ emission band series to be 1100-1700 K. The temperature of the plasma chamber wall was about $700 \mathrm{~K}$.

\subsection{CLASSIFICATION OF QCCS}

Three types of products were obtained from the ejected plasmic gases: gaseous products were obtained far from the orifice of the plasma chamber, black granular solid was collected on a substrate right in the ejected plasmic gases (granular QCC), and brown filmy matter was collected on the wall of the vacuum reaction chamber. They were analysed with mass spectrometer, electron microscope, X-ray diffraction, nuclear magnetic resonance (NMR), and electron spin resonance (ESR).

\subsubsection{Analysis of Produced Gases}

Quadrupole mass spectrometry showed that the gases produced were composed of $\mathrm{CH}_{4}, \mathrm{C}_{2} \mathrm{H}_{2}$, and unsaturated hydrocarbon molecules. The hydrocarbon molecules were a mixture of linear molecules, polyynes $(H-C \equiv C-C \equiv C \cdots C \equiv C-H)$, and ringed molecules such as benzene and its derivatives.

\subsubsection{Analysis of Granular QCC}

The granular QCC and filmy QCC were collected in the ejected plasma gas beam and were subject to the ultraviolet irradiation of the plasma. The granular QCC remained after treatment with methanol to remove filmy QCC.

Electron micrographs of the granular QCC showed that it is composed of grains of diameter size 45-50 nm. The X-ray diffraction pattern of granular QCC shows that it has a loosely-layered structure. The presence of free electrons in it was indicated by ESR measurements. 


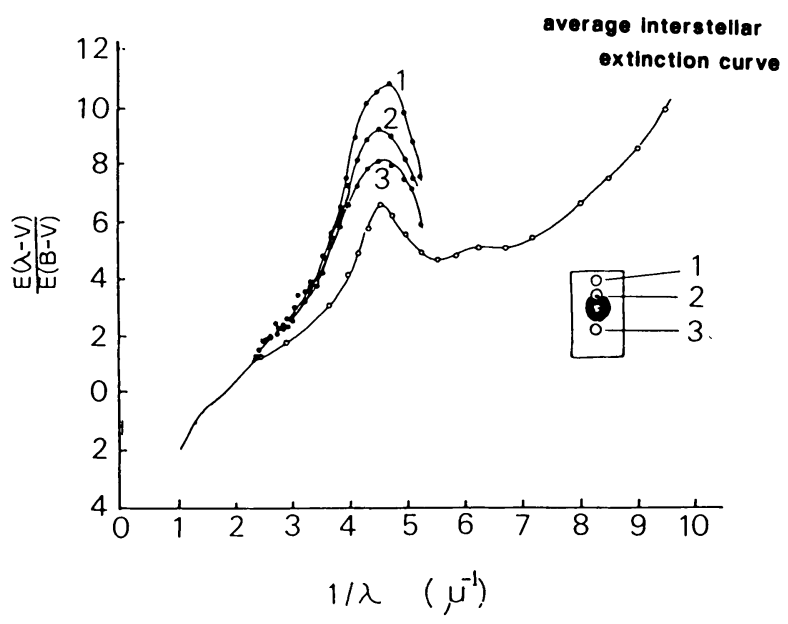

Fig. 1. Normalized extinction curves of the granular QCC (1-3) and the average interstellar extinction curve (open circles). The QCC was obtained on a quartz substrate $(30 \times 12 \mathrm{~mm})$ placed $50 \mathrm{~mm}$ from the orifice. The numerical values in the inset indicate the position of the measured point on the substrate.

\subsubsection{Analysis of Filmy QCC}

The brown, filmy QCC is soluble in methanol. This suggests that it is a mixture of low molecular-weight hydrocarbons. The NMR measurement showed that it contains four kinds of organic components such as polyynes, aromatics, olefins, and saturated hydrocarbon components. ESR measurements showed the presence of free electrons.

\subsection{OPTICAL AND INFRARED PROPERTIES OF QCC}

\subsubsection{Extinction Curve of Granular QCC in the Visual and UV Regions}

Granular QCC was obtained as a dark circular spot about $10 \mathrm{~mm}$ in diameter. The dark area was surrounded by light-brown material. Extinction of the QCC was measured in this area. Extinction curves for QCC were recalculated to give $\lambda^{-1}$ units on the abscissa, with $B=4400 \AA$ and $V=5500 \AA$ for normalization (see Fig. 1).

\subsubsection{IR Spectra of Filmy QCC}

In Fig. 2 we compare the IR spectrum of the filmy QCC to that of oxidized filmy QCC. Oxidation changes the spectrum of the filmy QCC in the following way:

- new features at 7.7 and $8.6 \mu \mathrm{m}$ clearly appear;

- the strength of the features at $6.2,7.3$, and $11.3 \mu \mathrm{m}$ are increased;

- a broad feature between 5.5 and $9.6 \mu \mathrm{m}$ appear; 


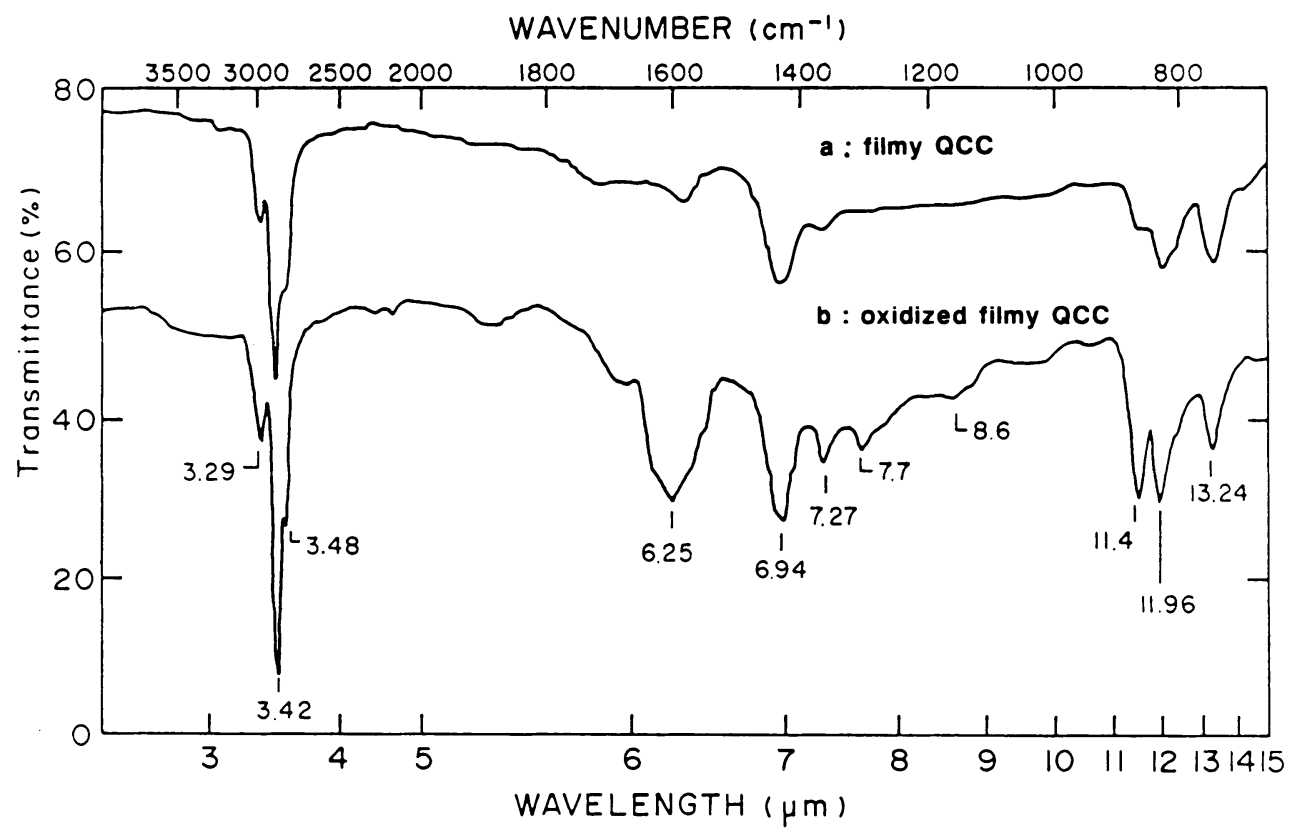

Fig. 2. Infrared spectra of unoxidized and oxidized filmy QCC (shifted by $\sim 20 \%$ in the ordinate).

- faint features are also seen at $5.3,9.7$, and $10.5 \mu \mathrm{m}$.

The IR spectrum of oxidized filmy QCC show a remarkable agreement with the UIR emission bands. Nine of the UIR bands $(3.3,3.4,3.5,6.2,6.9,7.7,8.6$, and $11.3 \mu \mathrm{m}$, and a plateau between 11.3 and $13.0 \mu \mathrm{m}$ ), as well as the broad feature between 5.5 and $9.6 \mu \mathrm{m}$ are evident in the spectrum.

The experimental results indicate that the features at 7.7 and $8.6 \mu \mathrm{m}$ in the oxidized filmy QCC result from the addition of oxygen.

Spectra of molecules such as 1, 4-naphthoquinone, benzophenone, and anthraquinone, clearly show bands at 7.7 and $8.6 \mu \mathrm{m}$. These molecules have the following special ketone structure in common:

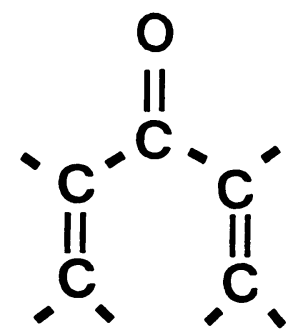

This is named a "cross-conjugated ketone" (CCK). 


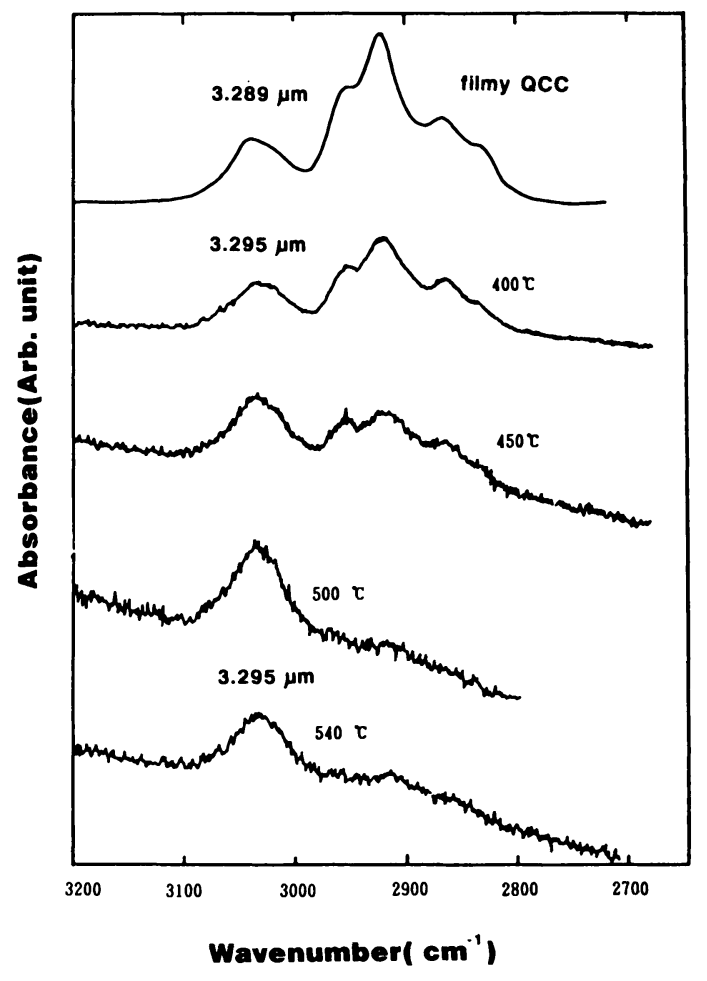

Fig. 3. Infrared spectral change of filmy QCC with a rise of temperature.

\subsubsection{IR Spectrum of Thermally Altered Filmy QCC}

After the filmy QCC was kept for 30 minutes at a given temperature under vacuum, IR spectra of the filmy QCC were measured (Fig. 3). Drastic changes in the spectra occurred between $250-350^{\circ} \mathrm{C}$. In this temperature range, the peak position of the $3.3 \mu \mathrm{m}$ feature was shifted slightly toward longer wavelengths: from $3.289 \mu \mathrm{m}$ to $3.295 \mu \mathrm{m}$. The strength of the 3.4 and $3.5 \mu \mathrm{m}$ features decreased between 300$350^{\circ} \mathrm{C}$. The structure of the filmy QCC was altered and fine granular particles were seen under electron microscope observations. Above $350^{\circ} \mathrm{C}$, the features at 3.295 and $11.4 \mu \mathrm{m}$ remained. This result suggests that the "special solo structure" of a single $H$ atom on a ring can exist at this temperature.

\section{CONCLUSION}

Characteristic spectral features measured in the observation of circumstellar and interstellar dusts and gases are seen in the spectra of the quenched or rapidly cooled matter from excited chemical species such as $C_{2}, C H$, and $H$. This suggests a process of dust formation in which these excited chemical species were cooled 
rapidly and solidified to form dusts in interstellar or circumstellar space.

\section{REFERENCES}

Sakata, A., Wada, S., Okutsu, Y., Shintani, H., and Nakada, Y. 1983, Nature, s01, 493.

Sakata, A., Wada, S., Tanabe, T., and Onaka, T. 1984, Ap. J. (Letters), 287, L51.

Sakata, A., Wada, S., Onaka, T., and Tokunaga, A. T. 1987, Ap. J. (Letters), 320, L63. 